\title{
Usos potenciais de óleos essenciais em remediação ambiental: Uma revisão
}

\author{
Potential uses of essential oils in environmental remediation: A review \\ Usos potenciales de los aceites esenciales en la remediación ambiental: Una revisión
}

Recebido: 16/05/2021 | Revisado: 23/05/2021 | Aceito: 29/05/2021 | Publicado: 12/06/2021

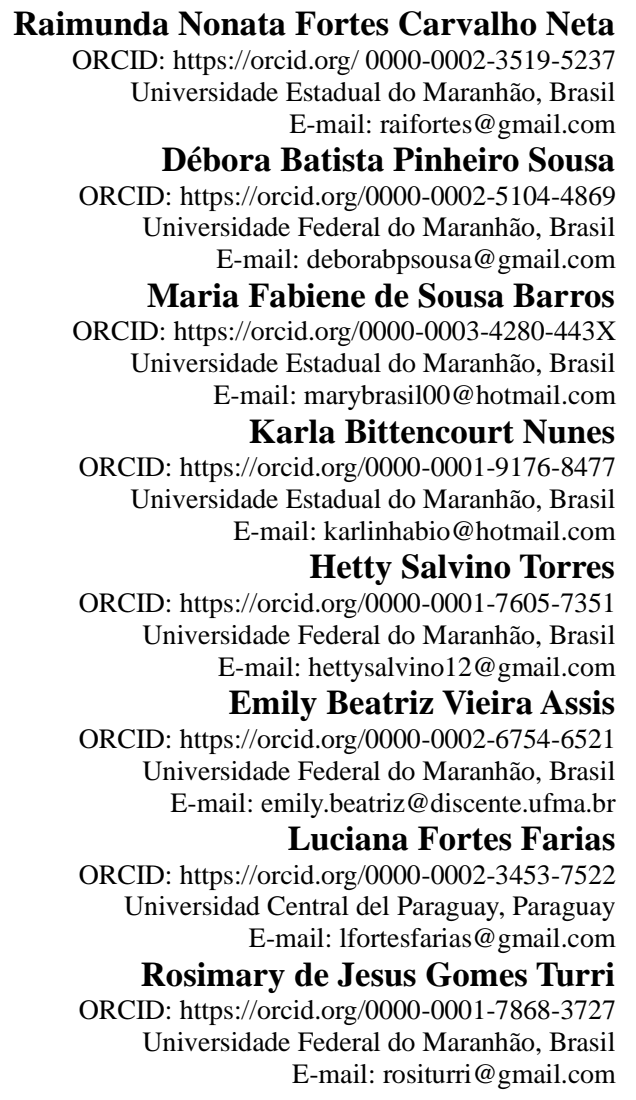

\section{Resumo}

Atualmente são pouco conhecidos os potenciais usos de óleos essenciais em contextos de áreas que passaram por processos de remediação ambiental. Neste trabalho objetivou-se realizar uma revisão sistemática sobre os potenciais usos dos óleos essenciais com aplicação em situações de remediação ambiental. A busca sistemática foi realizada em três bases de dados (Web of Science, PubMed e Scielo) com uso de descritores e operadores booleanos ("AND", "OR", "NOT"). Todos os resultados selecionados foram exportados a partir das bases de dados para análises posteriores no software livre VOSviewer, construindo-se as redes bibliométricas, mapeamento bibliométrico e representação gráfica dos mapas. O mapa geral de visualização de redes de co-ocorrência de termos indicou de 7 a 19 clusters com palavras-chave. Os óleos essenciais de Eucalipto, Limão Siciliano, Capim-Limão, Melaleuca, Cravo-daÍndia e Lavanda destacam-se como os mais utilizados nas pesquisas analisadas. As abordagens mais voltadas à remediação ambiental foram baseadas na análise de plantas aromáticas para remediar ambientes impactados por metais, destacando-se também a utilização dos óleos essenciais em estudos com inseticida/ controle de pragas (24\%), antimicrobiano contra patógenos ambientais (20\%), biorremediação de fungos (20\%) e fitorremediação de modo geral (17\%). As plantas aromáticas (das famílias Poaceae, Lamiaceae, Asteraceae e Geraniaceae) foram utilizadas nas pesquisas sobre remediação de locais contaminados porque agem como potenciais fitoestabilizadores, hiperacumuladores, bio-monitores e metalófitos facultativos. Esses dados indicam o potencial dos óleos essenciais (e das plantas aromáticas) em processos de remediação ambiental, sendo indicado seu uso em situações onde existem áreas que precisam ser remediadas e exigem intervenção imediata.

Palavras-chave: Fitorremediação; Biorremediação; Plantas aromáticas; Eucalipto; Melaleuca. 


\begin{abstract}
Currently, the potential uses of essential oils in contexts of areas that have undergone environmental remediation processes are little known. This work aimed to carry out a systematic review on the potential uses of essential oils with application in situations of environmental remediation. The systematic search was carried out in three databases (Web of Science, PubMed and Scielo) using descriptors and Boolean operators (“AND”, "OR", "NOT"). All selected results were exported from the databases for further analysis in the free software VOSviewer, building the bibliometric networks, bibliometric mapping and graphical representation of the maps. The general visualization map of term co-occurrence networks indicated 7 to 19 clusters with keywords. The essential oils of Eucalyptus, Sicilian Lemon, Lemongrass, Melaleuca, Cloves and Lavender stand out as the most used in the analyzed research. The approaches most focused on environmental remediation were based on the analysis of aromatic plants to remedy environments impacted by metals, highlighting also the use of essential oils in studies with insecticide / pest control (24\%), antimicrobial against environmental pathogens (20\%), fungal bioremediation (20\%) and phytoremediation in general (17\%). Aromatic plants (from the families Poaceae, Lamiaceae, Asteraceae and Geraniaceae) were used in research on remediation of contaminated sites because they act as potential phytostabilizers, hyperaccumulators, biomonitors and facultative metallophytes. These data indicate the potential of essential oils (and aromatic plants) in environmental remediation processes, indicating their use in situations where there are areas that need to be remedied and require immediate intervention.
\end{abstract}

Keywords: Phytoremediation; Bioremediation; Aromatic plants; Eucalyptus; Melaleuca.

\title{
Resumen
}

Actualmente, los usos potenciales de los aceites esenciales en contextos de áreas que han sufrido procesos de remediación ambiental son poco conocidos. Este trabajo tuvo como objetivo realizar una revisión sistemática sobre los usos potenciales de los aceites esenciales con aplicación en situaciones de remediación ambiental. La búsqueda sistemática se realizó en tres bases de datos (Web of Science, PubMed y Scielo) utilizando descriptores y operadores booleanos ("AND”, “OR", "NOT"). Todos los resultados seleccionados fueron exportados de las bases de datos para su posterior análisis en el software gratuito VOSviewer, construyendo las redes bibliométricas, el mapeo bibliométrico y la representación gráfica de los mapas. El mapa de visualización general de las redes de co-ocurrencia de términos indicó de 7 a 19 grupos con palabras clave. Los aceites esenciales de Eucalipto, Limón, Limoncillo, Melaleuca, Clavo y Lavanda se destacan como los más utilizados en las investigaciones analizadas. Los enfoques más específicos sobre remediación ambiental se basaron en el análisis de plantas aromáticas para remediar ambientes impactados por metales, destacando también el uso de aceites esenciales en estudios con insecticidas / control de plagas (24\%), antimicrobianos contra patógenos ambientales (20\%), biorremediación fúngica (20\%) y fitorremediación en general (17\%). Las plantas aromáticas (de las familias Poaceae, Lamiaceae, Asteraceae y Geraniaceae) se utilizaron en la investigación sobre la remediación de sitios contaminados porque actúan como potenciales fitoestabilizadores, hiperacumuladores, biomonitores y metalofitos facultativos. Estos datos indican el potencial de los aceites esenciales (y plantas aromáticas) en los procesos de remediación ambiental, indicando su uso en situaciones donde hay áreas que necesitan ser remediadas y requieren una intervención inmediata.

Palabras clave: Fitorremediación; Biorremediación; Plantas aromáticas; Eucalipto; Melaleuca.

\section{Introdução}

Remediação Ambiental é entendida como uma série de técnicas e operações para anular os efeitos adversos de elementos tóxicos (encontrados em determinado local) aos seres humanos e à biota em geral (Vinu \& Madras, 2010). Trata-se de um processo capaz de minimizar os danos causados pelos contaminantes e reduzir o impacto causado ao meio ambiente (Gonçalves Jr, 2013), sendo de grande relevância no contexto atual (Júnior et al., 2021), visto que existem muitas pessoas em todo o mundo vivendo em áreas que precisam ser remediadas (Ferreira et., 2020).

Dados de recente inventário realizado na União Europeia mostraram a existência de 648.964 áreas em que foram desenvolvidas atividades potencialmente contaminadoras do solo nos 28 países-membros, sendo que os poluentes mais encontrados foram metais pesados e óleos minerais (Pérez \& Eugênio, 2018). Para os Estados Unidos, os dados do estudo da Environmental Protection Agency (EPA), relataram que 77.000 áreas foram consideradas contaminadas, estimando-se que este número será de 294.000 em 2033, sendo que os poluentes mais frequentemente detectados nas áreas contaminadas norteamericanas, são principalmente metais, tais como arsênio, cromo, chumbo, zinco, níquel e cádmio (USEPA, 2004). No Brasil em 2014, o levantamento do SISSOLO (Sistema de Informação de Vigilância em Saúde de Populações Expostas a Solo Contaminado) mostrou a existência de cerca de 34 milhões de indivíduos expostos ou potencialmente expostos a toda gama de 
malefícios gerados pelas áreas contaminadas (Brasil, 2014). Dados do Ministério do Meio Ambiente, em 2020, apontam que no Brasil existe cerca de 117 áreas contaminadas por Poluentes Orgânicos Persistentes (POPs), especialmente na região sudeste (com cerca de $85 \%$ das áreas), sendo que as principais atividades causadoras de contaminação são a armazenagem, a disposição de resíduos e a produção industrial (MMA, 2020). Nessas regiões, as substâncias mais comumente encontradas são as bifenilas policloradas (PCBs), o hexaclorociclohexano $(\mathrm{HCH})$ e diclorodifeniltricloroetano (DDT) e o mercúrio (MMA, 2020). De um modo geral, os dados do Ministério da Saúde apontam que a atuação dos órgãos ambientais ocorre em apenas 33,8\% das áreas com populações expostas ou potencialmente expostas aos contaminantes (Brasil, 2014), fato que requer atenção de toda a sociedade e estudos que indiquem alternativas de remediação ambiental.

Nesse contexto, a remediação ambiental atua como um processo amplo, objetivando fazer a retirada da fonte geradora de determinada contaminação, a diminuição do nível de contaminação do solo (Marques et al., 2011), da água, do ar para níveis aceitáveis pela legislação dos diferentes países (Brito \& Vasconcelos, 2012), bem como reduzir os riscos ambientais ou de exposição de profissionais que trabalham na área e/ou usuários do local e do recurso, a partir do desenvolvimento e emprego de tecnologias diversas (Júnior et al., 2021).

Existem várias técnicas tradicionais de remediação ambiental que podem ser executadas em diferentes regiões contaminadas, podendo-se citar: a) atenuação natural, que é baseada em processos destrutivos e não-destrutivos dos contaminantes encontrados na área; b) barreiras de água, que visam impedir que determinada contaminação avance, por meio de barreiras feitas com trincheiras ou poços diretamente localizados no fluxo contaminante; c) barreiras reativas, onde o material reativo coletado é inserido diretamente dentro do aquífero, de tal forma que seja atravessado pela água; d) Encapsulamento geotécnico, no qual se promove o isolamento do local contaminado, por meio de barreiras e coberturas; e) Biorremediação, que envolve o uso de microrganismos (bactérias e fungos) para executar a transformação de substâncias, diminuindo ou extraindo totalmente fatores tóxicos delas; f) Fitorremediação, que envolve o uso de plantas para a redução dos teores de contaminantes a níveis seguros (Vidali, 2001; Arthur et al., 2005; Biondi et al., 2011; Adams et al., 2015; Rodrigues \& Orlandelli, 2018). Além da aplicação dessas técnicas, tradicionalmente tem sido muito utilizada a incineração (destruição térmica realizada sob altas temperaturas de até $1.250^{\circ} \mathrm{C}$ ) para a extração de compostos orgânicos voláteis e semi-voláteis (como PAHs, PCBs, pesticidas), mas que pode ocasionar emissões de substâncias tóxicas capazes de poluir a atmosfera, a água e o solo e com efeitos nocivos para a saúde humana (Vidali, 2001). Assim, o próprio processo de remediação pode gerar efeitos nocivos, especialmente problemas respiratórios para a população direta ou indiretamente envolvida com a área contaminada.

No processo de remediação ambiental é preciso atuar também nos aspectos que envolvem a saúde ocupacional e das pessoas envolvidas com a área. Análises diversas indicam que milhões de idosos e crianças são fortemente afetados em áreas contaminadas, necessitando-se, portanto, de ações voltadas para a saúde dessas pessoas (USGAO, 2015). Nesse sentido, os óleos essenciais podem ser utilizados para auxiliar na saúde e bem-estar, especialmente, em afecções do sistema respiratório, gastrointestinal, sistema nervoso central, sistema circulatório e cardíaco, entre outras patologias humanas (Bayala et al., 2014). Contudo, ainda são poucos os estudos que abordam a remediação ambiental em um contexto tão amplo. Além disso, apesar do uso disseminado de muitas plantas para fazer remediação ambiental, no chamado processo de fitorremediação (Rodrigues \& Orlandelli, 2018), ainda são poucos os estudos que abordam usos dos óleos essenciais aplicados a situações de remediação ambiental.

Os óleos essenciais podem ser definidos como compostos sintetizados e armazenados em tricomas glandulares de plantas aromáticas, voláteis e complexos, que na maioria, são formados principalmente de mono e sesquiterpenos e de fenilpropanóides (Bizzo, 2009), caracterizados por um forte odor, sendo sintetizados durante o metabolismo secundário do vegetal (Machado \& Junior, 2011) e envolvendo múltiplos compostos químicos com diferentes aplicações em saúde e 
ambiente (Tian et al., 2020). A indústria dos óleos essenciais tem se expandido muito nos últimos anos, existindo muitas aplicações desses produtos como inseticidas, antifúngicos, antibacterianos, antivirais, entre outros (Knaak \& Fiuza, 2010; Contrucci et al., 2019). Todavia, ainda pouco se conhece sobre as aplicações dos óleos essenciais em contextos de áreas que passaram por processos de remediação ambiental. Assim, nesse trabalho objetivou-se realizar uma revisão sistemática sobre os potenciais usos dos óleos essenciais com aplicação em situações de remediação ambiental.

\section{Metodologia}

A busca sistemática foi realizada em três bases de dados: Web of Science, PubMed e Scielo. Foram utilizados os descritores essential oil (óleos essenciais), environment (Meio ambiente), environmental remediation (remediação ambiental), bioremediation (biorremediação), phytoremediation (fitorremediação). A busca nessas plataformas foi efetuada por meio de descritores (ou palavras-chave) com auxílio dos operadores booleanos: "AND", "OR", "NOT”. O período abordado envolveu os anos de 2010 a 2021. Foram aplicados critérios de elegibilidade, onde se considerou apenas trabalhos publicados do tipo artigo, bem como critérios de exclusão, onde não foram considerados os trabalhos do tipo artigos de revisão, textos avulsos, monografia, dissertação, tese e publicações em anais. Além disso, durante as buscas, o título e o resumo dos artigos foram previamente analisados para ponderar se os mesmos apresentavam informações na temática de interesse (estudos com óleos essenciais e aplicações em situações de remediação ambiental). Todos os resultados selecionados foram exportados a partir das bases de dados para análises posteriores no software VOSviewer (versão 1.6.15 www.vosviewer.com), construindo-se as redes bibliométricas, mapeamento bibliométrico e representação gráfica dos mapas. A criação das redes foi feita a partir da funcionalidade de mineração de textos, que permite visualizar e construir redes de co-ocorrência de termos importantes e extraídos do corpo dos textos científicos. Na elaboração dos mapas são utilizados algoritmos que medem a distância entre dois termos, que refletem a força de relação entre os itens, sendo que uma pequena distância indica uma forte relação entre os termos; e quanto maior a distância, menos relacionados estão os termos (Vaneck \& Waltman, 2010).

Todos os manuscritos que passaram nos filtros foram baixados e salvos em uma pasta online para posterior exclusão das repetições. Em seguida os manuscritos foram analisados na íntegra e os dados tabelados para obter informações de autores, ano, título, objetivo da pesquisa, país, principais óleos essenciais analisados, qual a planta utilizada, principais compostos do óleo essencial, finalidade do estudo, além da área de aplicação do óleo essencial (se ambiente ou saúde).

\section{Resultados e Discussão}

O resultado da busca sistemática iniciou com 1078 artigos para a base de dados Web of Science, entretanto apenas 127 estavam relacionados com o objetivo da pesquisa. Para a base Scielo a pesquisa iniciou com 888 trabalhos, onde apenas 55 estavam no período estabelecido. Na base PubMed a primeira busca recuperou 2500 publicações, onde 127 artigos foram utilizados após a triagem.

O mapa geral de visualização de redes de co-ocorrência de termos (Figura 1) indicou de 7 a 19 clusters com palavraschave para os resultados das três bases de dados analisadas (Web of Science, Scielo e PubMed). Na base Web of Science observou-se que o maior grau de relacionamento dos termos ocorreu no Cluster 1 (vermelho) que apresentou 7 itens, tais como antibacteriano, antifúngico, antioxidante, citotóxico e agricultura sustentável. A maioria das publicações focam o papel dos óleos essenciais na saúde humana, especialmente, como antifúngicos, antibacterianos, antivirais, mas também como uma fonte importante de fungicidas sustentáveis para o tratamento de frutas e vegetais contra patógenos pós-colheita (Jiménez-Reyes et al., 2019), auxiliando também na produção sustentável dos chamados "produtos orgânicos" (Pellegrini et al., 2018). Na base de dados Scielo, observou-se que o maior grau de relacionamento dos termos ocorreu no Cluster 1 (vermelho) que apresentou 14 itens, destacando-se os descritores influência ambiental, extratos de plantas, nematoides, pesticidas, polinizadores e fungos de 
solo, entre outros. Os trabalhos de um modo geral destacam o papel dos óleos essenciais como pesticidas sustentáveis, sendo muitas as pesquisas que apontam a importância de se substituir compostos químicos tradicionais usados na agricultura pelos ósseos essenciais, enquanto alternativas naturais mais seguras para eliminar fungos (Soylu et al., 2010; Vitoratos et al., 2013; Bouyahya et al., 2016), insetos (Russo et al., 2018) e outras pragas. Na base PubMed verificou-se que o maior grau de relacionamento dos termos ocorreu no Cluster 1 (vermelho) que apresentou 39 itens, envolvendo especialmente termos relacionados com biodegradação, bactérias, restauração ambiental, biodiversidade, poluição da água, fitoestabilizador, solo, entre outros. Os trabalhos focam em óleos essenciais e plantas aromáticas para biorremediação de fungos residenciais e ocupacionais (Rogawansamy et al., 2015) e fitorremediação de solos contaminados (Jain et al., 2020)

Figura 1. Mapa de visualização de redes de co-ocorrência de termos, gerado a partir dos dados globais extraídos das bases de dados: A) Web of Science; B) Scielo; C) PubMed.

A) Web of Science

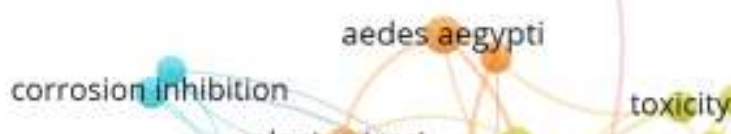

plant extract

origanum vulgare

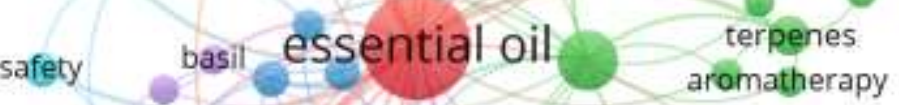

carvacrol antimicrobial activity

antifungal

secondary metabolites

antibacterial

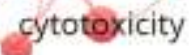


B) Scielo

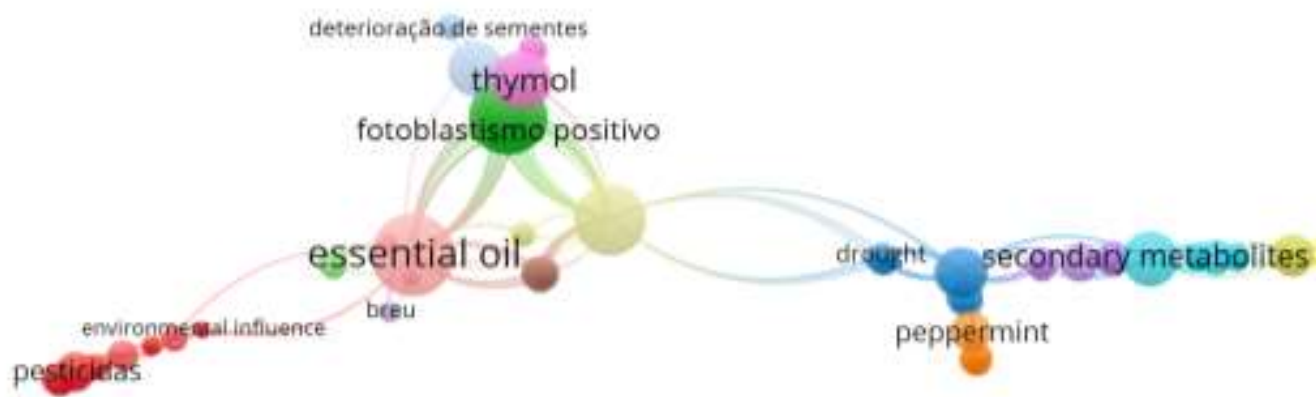

C) PubMed

\section{VOSviewer}

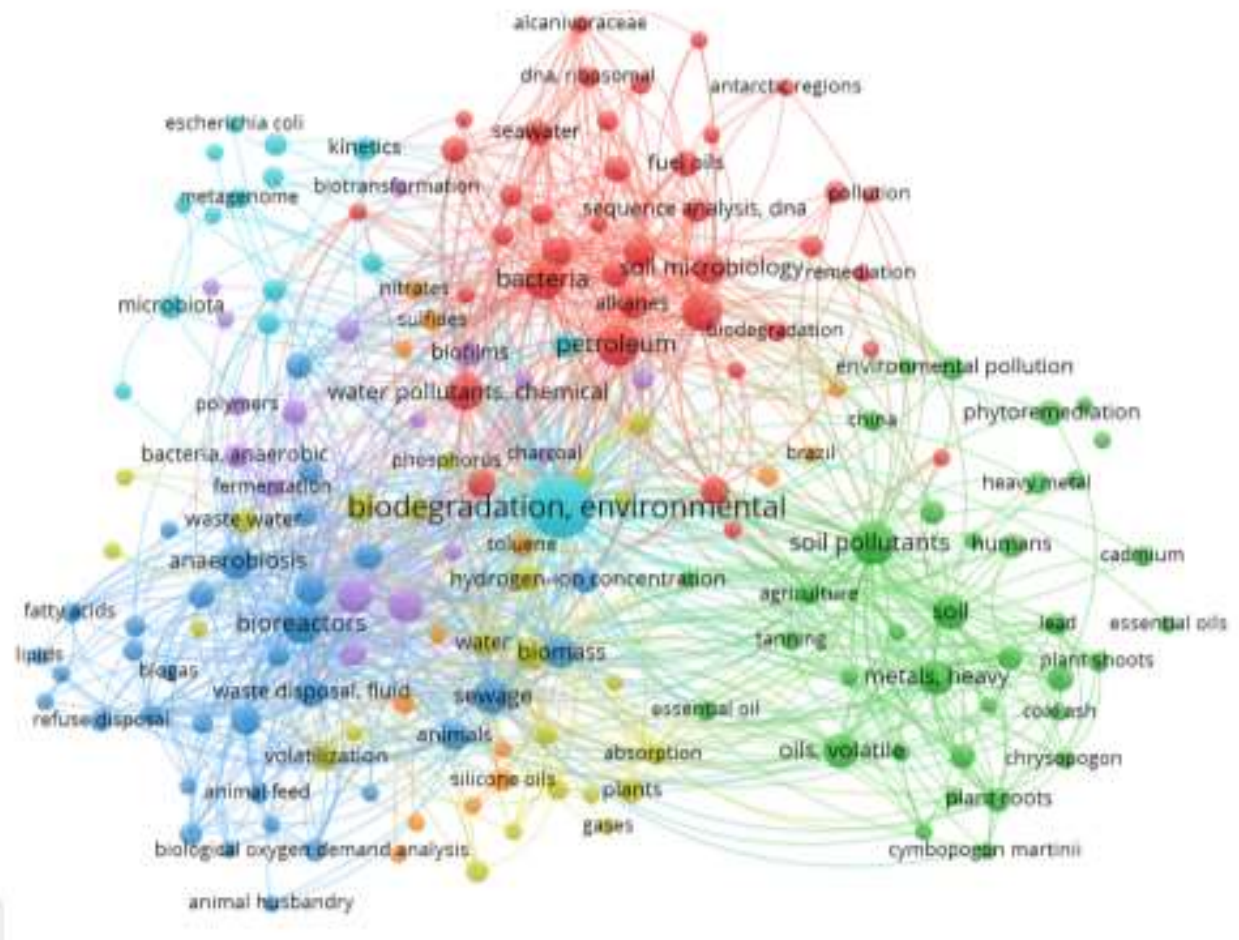

Fonte: Autores.

A Figura 2 mostra a quantidade de publicações no período de busca (2010-2021) para todas as bases de dados. Desde 2010, as pesquisas acerca da utilização de óleos essenciais em remediação ambiental (e em outras áreas relacionadas a meio ambiente) aumentaram, chegando a triplicar no período compreendido entre os anos de 2019-2020. Esses dados mostram que essa é uma temática que merece atenção da comunidade científica e que tem recebido investimento de agências de fomento. 
Figura 2. Número de publicações por período de busca (2010-2021) nas bases de dados Web of Science, Scielo e PubMed.

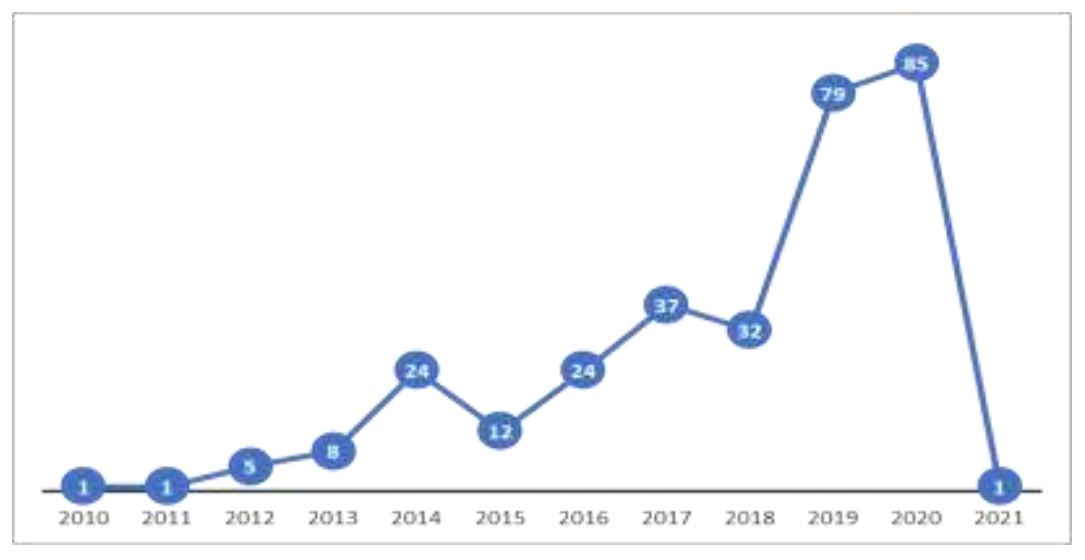

Fonte: Autores.

Em relação aos países e/ou nações que realizam pesquisas com óleos essenciais na temática em questão, a Índia, Brasil e Itália destacam-se com iniciativas nessas temáticas (Figura 3). A Índia tem lugar de destaque na produção e tradicional uso de óleos essenciais (Sangwan et al., 2001). O Brasil é considerado um grande produtor e exportador mundial de óleos essenciais, vendendo, especialmente para a União Europeia, óleos essenciais de limão, lima e laranja e outros cítricos (Bizzo et al., 2009). As pesquisas sobre os óleos essenciais nesses países, de um modo geral, estão focadas nos óleos essenciais mais vendidos no mercado mundial.

Figura 3. Países/nações que se destacam na pesquisa com óleos essenciais, com foco em remediação ambiental, no período de 2010-2021, nas bases de dados Web of Science, Scielo e PubMed.

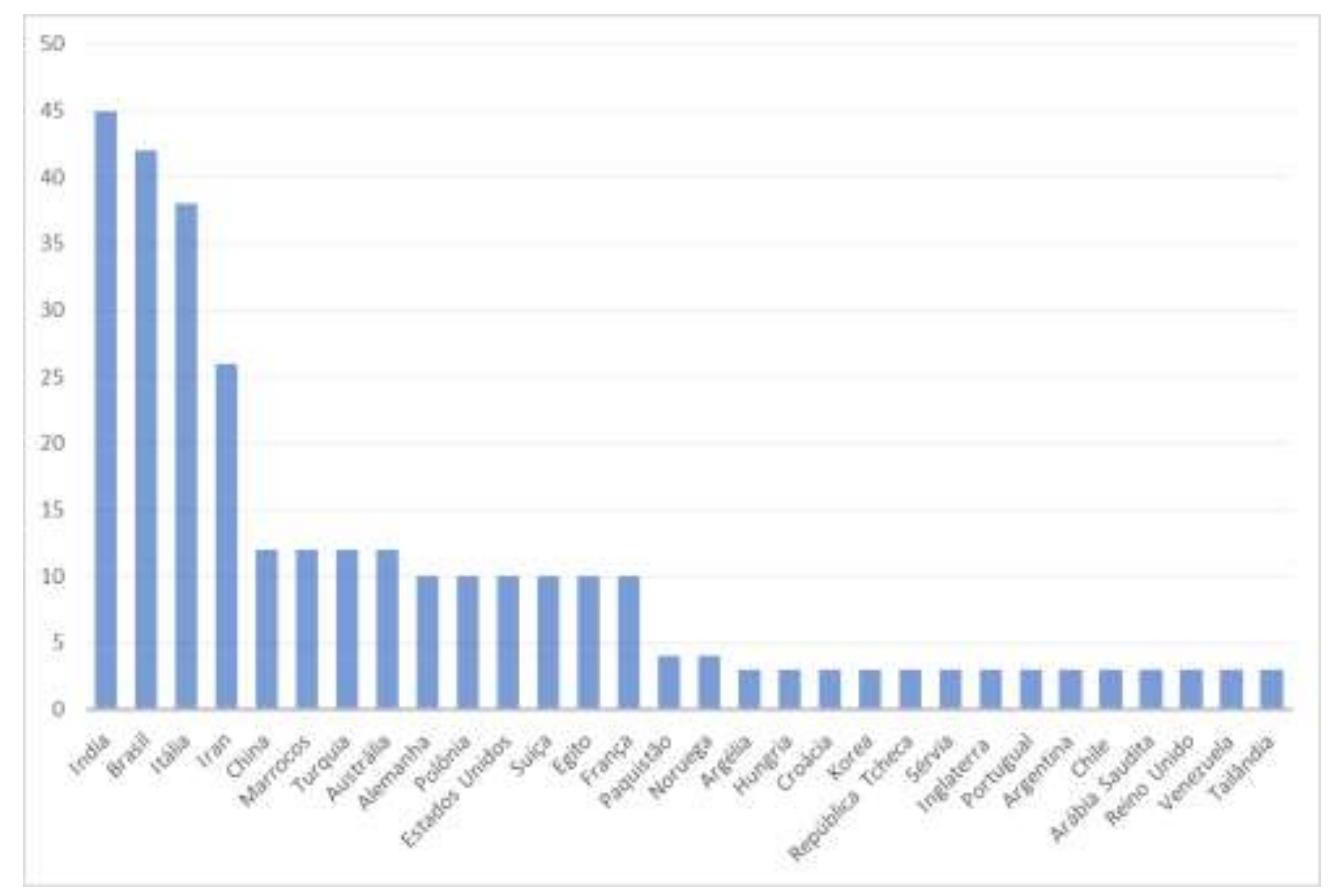

Fonte: Autores.

Os óleos essenciais de Eucalipto, Lemon (Limão Siciliano), Lemongrass (Capim-Limão), Melaleuca, Cravo-da-Índia e Lavanda destacam-se como óleos essenciais mais utilizados nas pesquisas das publicações analisadas (Figura 4). O óleo essencial de Eucalipto (Eucalyptus globulus e Eucalyptus radiata) é extraído por destilação a vapor de folhas e galhos, apresentando vários componentes químicos (cimeno, cineole, globulol, limoneno, pineno, pinocaveol, cariofileno, geraniol, 
piperitona, entre outros) que podem ser usados na assepsia ambiental e em casos de problemas respiratórios (Castro \& Lima, 2010). Já o Lemon ou Limão Siciliano (Citrus limon) é um óleo essencial extraído pela prensagem a frio da casca da fruta que apresenta muitos componentes químicos (acetato de nerila, cimeno, geraniol, limoneno, mirceno, neral, pineno, sabineno, terpineol entre outros) utilizados para desintoxicação de pessoas e purificação de ambientes (Martins et al., 2017).

Figura 4. Tipos de óleos essenciais utilizados nas investigações publicadas, no período de 2010-2021, nas bases de dados Web of Science, Scielo e PubMed.

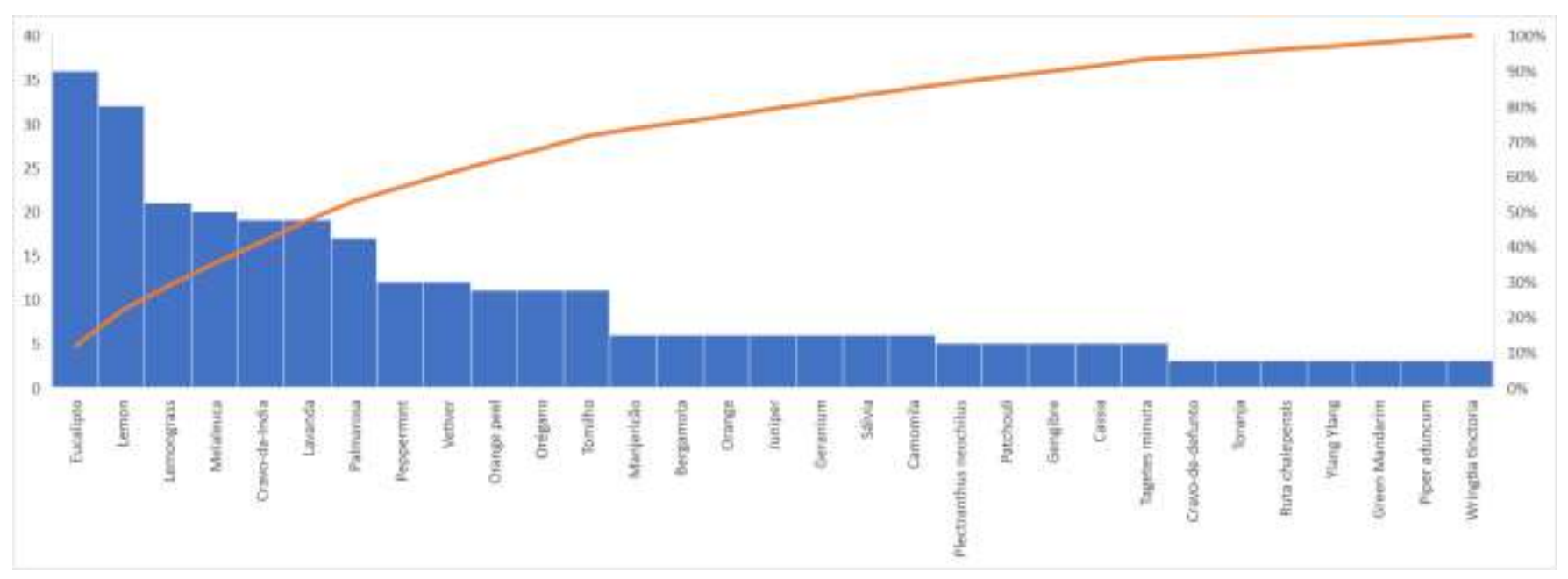

Fonte: Autores.

O Lemongrass ou Capim-Limão (Cymbopogon citratus) tem seu óleo essencial extraído por destilação a vapor das folhas (frescas ou parcialmente secas) da planta e apresenta componentes químicos (acetato de geranila, citronelal, geraniol, mirceno, neral com traços de limoneno e citral, entre outros) que têm sido usados, principalmente, para matar germes, repelente de insetos e desodorizante do ar (Pinto et al., 2014). Esse óleo produzido no Brasil é constituído de 60 a $80 \%$ de citral (mistura de geranial e neral) que é matéria-prima relativamente barata para obtenção de produtos com maior valor comercial agregado (Craveiro \& Queiroz, 1993).

A Melaleuca ou Tea tree (Melaleuca alternifolia) é uma planta utilizada tradicionalmente e tem seu óleo essencial extraído por destilação a vapor das suas folhas, apresentando vários componentes químicos (aromadendreno, cadineno, cimeno, cineole, globulol, ledeno, limoneno, pineno, sabineno, terpineol, viridiflorol, entre outros) com fortes propriedades antissépticas e usado para combater infecções e fungos (Puvača et al., 2020). Os estudos sobre os mecanismos de ação antimicrobiana do óleo essencial de Melaleuca indicam que esse óleo essencial causa lise e perda da integridade da membrana das bactérias, devido à saída de íons e inibição da respiração (Carson et al., 2006). Diversos estudos mostram a redução do número de bactérias, quando linhagens foram submetidas aos vapores de óleos essenciais, durante a desinfecção de ambientes fechados, bem como nos sistemas de ventilação (Pibiri et al., 2006; Puvača et al., 2020).

O óleo essencial de Cravo-da-Índia (Eugenia caryophyllata) é extraído por destilação a vapor de folhas da planta e apresenta muitos compostos químicos (acetato de cariofileno, eugenila, eugenol, isoeugenol) que têm sido utilizados para livrar a atmosfera de doenças infecciosas, já que possui propriedades terapêuticas analgésicas, anestésicas, antibactericidas, antifúngicas, antissépticas, entre outras (Affonso et al., 2012).

A Lavanda (Lavandula angustifolia) é uma planta muito conhecida mundialmente e tem seu óleo essencial obtido através da destilação a vapor das suas flores, apresentando vários componentes químicos (acetato de geranila, acetato de linalila, acetato de octen-3-ila, cânfora, cariofileno, farneseno, linalol, terpineno, entre outros) muito utilizados para 
afugentamento de insetos e primeiros socorros em casos de queimaduras e ferimentos, já que tem várias propriedades terapêuticas, tais como antisséptico, antitóxico, antivenenoso, desintoxicante, anticoagulante, entre outros (Santos et al., 2019).

Todos os óleos essenciais são conservantes, sendo esse fato de grande interesse para as indústrias alimentícias, farmacêuticas e cosméticas, especialmente desde que o uso de aditivos naturais ganhou importância como tendência na substituição de conservantes sintéticos artificiais (Okoh et al., 2010). Nesse sentido, os OEs são bons candidatos para aplicação potencial como biopreservativos em alimentos e / ou ambientes de fabricação de alimentos (Pellegrini et al., 2018).

Em relação a aplicação dos óleos essenciais, a figura 5 mostra as principais abordagens realizadas e que constam nas publicações analisadas, destacando-se a utilização dos OEs para estudos com inseticida/ controle de pragas (24\%), antimicrobiano contra patógenos ambientais (20\%), biorremediação de fungos (20\%) e fitorremediação (17\%). As abordagens mais voltadas à remediação ambiental em sentido mais stricto sensu (biorremediação, fitorremediação e outras técnicas) foram baseadas especialmente na análise de plantas aromáticas para remediar ambientes impactados por metais, bem como como fitoestabilizadores de solo, tratamento de solo contaminado com lodo de curtume e de minas de carvão.

Figura 5. Percentual das principais aplicações dos óleos essenciais utilizados nas investigações publicadas e analisadas (período de 2010-2021) nas bases de dados Web of Science, Scielo e PubMed.

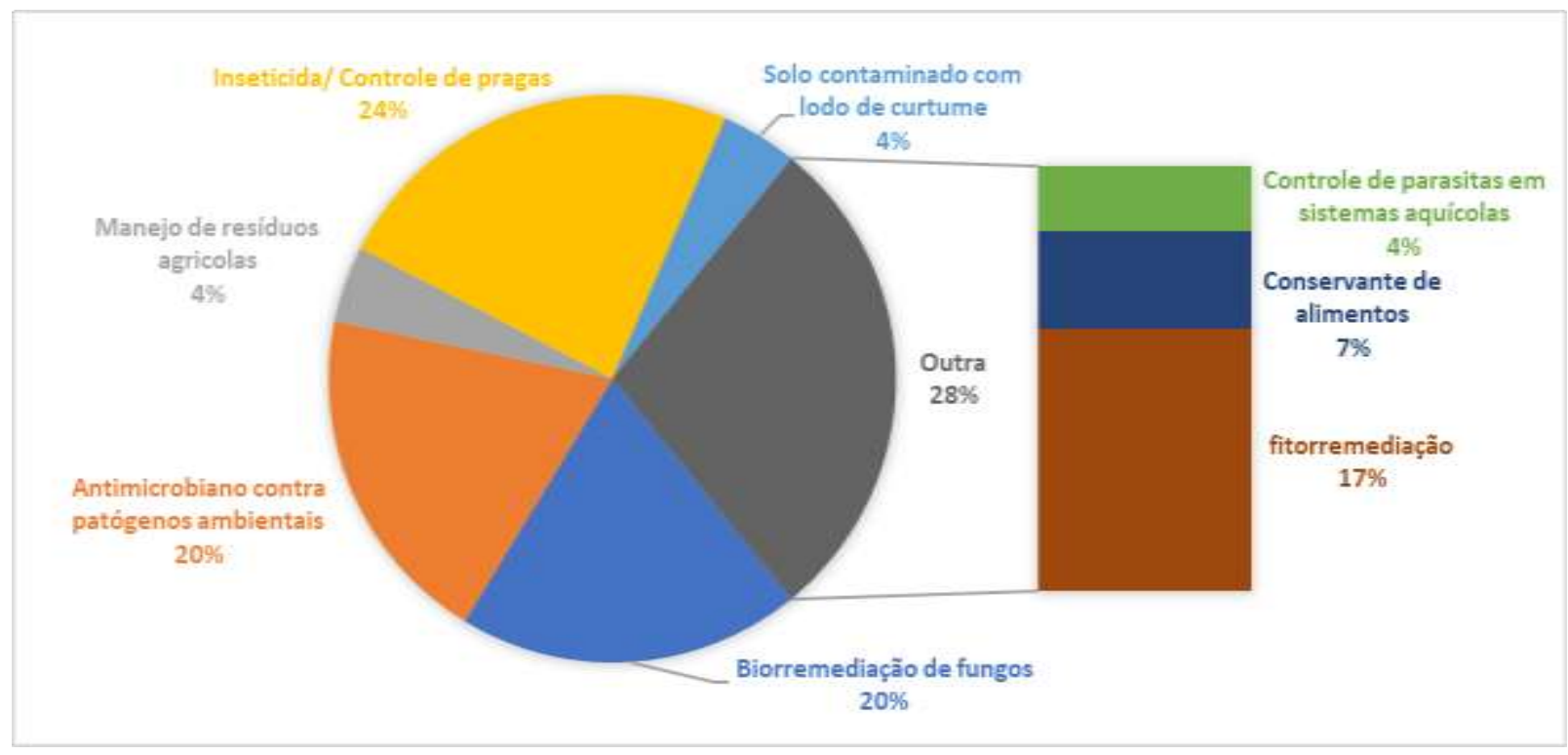

Fonte: Autores.

$\mathrm{Na}$ área ambiental muito se tem comentado sobre o controle de pragas (insetos diversos, ácaros e outros) de uma forma ecologicamente correta com uso de óleos essenciais (Chagas et al., 2002; Volpe et al., 2016). Os óleos essenciais interferem no sistema nervoso octopaminérgico dos insetos (mas não de mamíferos) e atuam de forma segura para os humanos, sendo investigados por terem ação como repelente, impedimento de oviposição, atividades regulatórias e antivetoras de crescimento (Koul et al., 2008). As abordagens das publicações são voltadas especialmente para: o recomendado potencial de uso de óleo essencial de eucalipto (Eucalyptus citriodora) para o controle de cupim subterrâneo Coptotermes gestroi (considerada uma das principais espécies-praga de ambiente urbano no Brasil) em cujo controle tradicional tem sido utilizados inseticidas químicos com alto riscos de intoxicação da população, contaminação do ambiente e dificuldade de isolamento de áreas tratadas (Mikola et al., 2017); uso de óleo essencial de Ruta chalepensis como alternativa saudável no combate ao Tribolium castaneum (uma praga mundial de grãos) que tem sido combatida tradicionalmente com pesticidas químicos que têm efeitos adversos na saúde e no meio ambiente (Najem et al., 2020); atividade larvicida dos óleos essenciais de Ocimum 
campechianum, Ocotea quixos e Piper aduncum contra Aedes aegypti (principal vetor dos vírus da febre amarela, dengue, chikungunya e zika), indicando-se a possibilidade de cultivo local dessas plantas em áreas tropicais e subtropicais e o preparo e produção em larga escala do OE das plantas nessas regiões para o controle desses vetores ainda na fase larval (Scalvenzi et al., 2019); eficácia do óleo essencial de Piper aduncum contra ninfas e adultos de Diaphorina citri (o inseto vetor das bactérias que causam o "grenning", a principal doença que afeta a citricultura), sendo considerado um produto de baixa toxicidade alternativo para os tradicionais produtos utilizados que causam contaminação ambiental, tem impacto negativo nos organismos benéficos e leva à resistência aos psilídeos (Volpe et al., 2016). Esses e outros dados indicam que os óleos essenciais são relativamente não tóxicos para mamíferos e atendem aos critérios de pesticidas de "risco reduzido", podendo trazer benefícios diversos para a agricultura em diversas situações, particularmente para a produção de alimentos orgânicos (Koul et al., 2008).

As publicações analisadas, cujas análises utilizaram os óleos essenciais para a remediação de fungos, foram muitas e diversificadas, como por exemplo: comparação de formulações comerciais e óleo de Melaleuca (Melaleuca alternifolia) para remediação de fungos (Aspergillus fumigatus e Penicillium chrysogenum) do ar de ambientes internos, sendo óleo essencial o agente antifúngico mais eficaz testado, podendo ter aplicação industrial na remediação de contaminação por fungos em edifícios residenciais e ocupacionais (Rogawansamy et al., 2015); testes com óleos essenciais de plantas ecologicamente corretas quanto à sua eficiência na proteção de madeiras valiosas contra a colonização por mofo e fungos da decomposição da madeira (Aspergillus niger, Penicillium commune, Coniophora puteana, Trametes versicolor, Chaetomium globosum) e infecção natural por esporos transportados pelo ar (Bahmani \& Schmidt, 2018); uso de óleo essencial de orégano na prevenção da infecção por Plasmopara vitícola (principal patógeno da viticultura mundial) em videiras (Vitis Vinifera) e estimulante dos mecanismos de imunidade das plantas, sendo indicado com alternativa para a redução de fungicidas sintéticos na agricultura (Rienth et al., 2019).

Nos artigos analisados, as abordagens sobre óleos essenciais como antimicrobiano contra patógenos também foram bem diversas, tais como: avaliação da atividade antimicrobiana de óleos essenciais de plantas medicinais (Ricinus communis, Sesamum indicum, Madhuca longifólia, Eclipta prostrata, Alternanthera sessile, Hydnocarpus pentandra, Wringtia tinctoria, Nigella sativa, Azadirachta indica) contra Pseudomonas isoladas de ambientes aquáticos, sendo W. tinctoria indicado como significativo para aplicação em remediação de sistemas de aquicultura (Vaseeharan et al., 2013); mistura do EO de laranja, patchuli, hortelã-pimenta e sálvia como sendo benéfico para a inibição do crescimento de vários microrganismos (Staphylococcus aureus, Streptococcus pneumoniae, Pseudonomas aeruginosa e Aspergillus brasiliensis), fornecendo uma opção antimicrobiana natural sobre fragrâncias sintéticas usadas em sabonetes, sprays corporais e purificadores de ar (VieiraBrock et al., 2017); uso de mistura de óleos essenciais de orégano, canela e cravo como potencial desinfetante em várias áreas, observando-se a redução do número de bactérias aeróbias mesófilas totais e Escherichia coli abaixo dos limites de detecção, sendo essa sinergia de óleos essenciais indicadas como alternativa ao etanol, o qual é comumente utilizado para a desinfecção de ambientes, equipamentos e superfícies (Sengun et al., 2020); uso de OE da casca de canela como ativo contra cepas clínicas e ambientais de Acinetobacter baumannii, sendo esse óleo indicado para ser empregado no combate a infecções causadas por bactérias do gênero Acinetobacter como componentes de formulações para higiene e desinfecção de ambiente hospitalar (Sienkiewicz et al., 2014).

$\mathrm{Na}$ fitorremediação as investigações analisadas foram voltadas especialmente para uso de plantas aromáticas utilizadas em tratamento de solos contaminados por metais e análises da segurança dos óleos essenciais produzidos em tais circunstâncias. As principais abordagens identificadas foram: análises de manjericão (Ocimum basilicum) para fitorremediação de áreas contaminadas por metais pesados (cádmio e chumbo) em áreas urbanas, mostrando-se que o solo contaminado foi fitorremediado e ocorreu o aumento o rendimento de EOs (Youssef, 2021); verificação das alterações dos compostos voláteis e do óleo essencial concentrado de Plectranthus neochilus (planta aromática) após a fitorremediação do herbicida ácido 2,4- 
diclorofenoxiacético (2,4-D) e a consequente atividade antibacteriana desse óleo essencial, mostrando-se que a exposição ao 2,4-D não causou muitas alterações nos compostos voláteis, nem no concentrado do óleo essencial da planta, sendo que o óleo essencial continuava adequado para ser usado normalmente como antimicrobiano (Ramborger et al., 2021); revegetação de solos contaminados com chumbo usando a planta Vetiver (Chrysopogon zizanioides) que pode concentrar o metal em suas raízes e ainda produzir OE em grandes quantidades (Danh et al., 2011); fitorremediação com a plantação de Palmarosa (Cymbopogon martini) para corrigir o solo ácido e resíduos de mina com biocarvão, sendo destacado que plantação de Palmarosa oferece uma tecnologia sustentável para fitoestabilização de resíduos de minas, juntamente com a produção de óleo essencial industrialmente importante (Jain et al., 2020); uso de Capim-limão (Cymbopogon citratus) na fitoestabilização de composto oriundo de lixão, sendo que a planta reduziu a concentração de metais do composto e produziu uma grande quantidade de óleo essencial com uma baixa concentração de metais considerado seguro para uso humano (Ultra, 2020); constatação de que o tratamento de lodo de curtume com Tagetes minuta (planta aromática da família Asteraceae) aumentou o seu crescimento e a produção de óleo da planta e também a atividade da urease e do nitrogênio da biomassa microbiana do solo (Patel \& Patra, 2014); análise de lodo de curtume como material de correção do solo e Palmarosa (Cymbopogon martinii) como potencial fitostabilizador, observando-se que ocorreu aumento da produtividade da cultura e o acúmulo de metal ocorreu nas raízes com uma translocação escassa para os brotos e o produto (óleo essencial) que é extraído por hidrodestilação (sem chance de contaminação do óleo), sendo o óleo comercialmente aceitável (Pandey et al., 2015).

Análises integradas têm mostrado que a fitorremediação de áreas contaminadas por metais não é indicada com o uso de culturas (plantas) comestíveis, pois os metais podem entrar na cadeia alimentar, mas as plantas aromáticas, de enraizamento profundo e estabilizadoras de metais com alta biomassa, que podem produzir produtos de alto valor, têm um grande potencial (Elallem et al., 2020). A técnica de fitorremediação com plantas aromáticas (produtoras de óleos essenciais) apresenta-se muito promissora, uma vez que faz a descontaminação com menos custo, sendo mais bem aceita por parte da população e não provoca contaminação secundária (Vasconcelos et al, 2012; Pandey et al., 2015). As plantas aromáticas (especialmente das famílias Poaceae, Lamiaceae, Asteraceae e Geraniaceae) podem ser usadas para a remediação de locais contaminados, especialmente com metais pesados, pois são culturas não alimentares, e que agem como potenciais fitoestabilizadores, hiperacumuladores, biomonitores e metalófitos facultativos (Pandey et al., 2019). Esses dados indicam o potencial das plantas aromáticas e dos óleos essenciais em processos de remediação ambiental, sendo necessárias mais investigações nessa temática em países onde as áreas que precisam ser remediadas exigem intervenção imediata.

\section{Considerações Finais}

As redes de co-ocorrência de termos formadas a partir dos artigos revisados no presente trabalho mostram que muitas pesquisas apresentam resultados significativos para o contexto da remediação ambiental, especialmente sobre o poder dos óleos essenciais enquanto agentes antibacterianos, antifúngicos e citotóxicos, sendo capazes de auxiliar em processos de saúde física de trabalhados e remoção de substâncias e microrganismos de ambientes contaminados. Contudo, esses trabalhos não usam diretamente a terminologia remediação ambiental e ainda são poucas as correlações existentes com a temática de modo mais amplo.

Os dados desta revisão mostram que pesquisadores de países com economias que exportam ou importam óleos essenciais (tais como Índia, Brasil, Itália, China, Marrocos, Turquia e Austrália) têm investido em estudos sobre o potencial dos óleos essenciais para aplicações que vão além da saúde humana, envolvendo aspectos de responsabilidade ambiental e, consequentemente, aplicações em situações de remediação ambiental.

Nesse contexto, os óleos essenciais (e suas plantas aromáticas de origem) surgem como aliados para remediar áreas contaminadas e auxiliar na saúde de pessoas que trabalham e vivem nessas áreas, apresentando uma maior eficiência nas suas 
aplicabilidades, oferecendo mais segurança para o meio ambiente e para a saúde humana.

\section{Agradecimentos}

Agradecemos à equipe do Laboratório de Biomarcadores em Organismos Aquáticos da Universidade Estadual do Maranhão (UEMA) pelo suporte nas análises estatísticas e à Gláucia Miralha e ao Luciano Miralha pelas valiosas informações sobre óleos essenciais.

\section{Referências}

Adams, G. O., Fufeyin, P. T., Okoro, S. E., \& Ehinomen, I. (2015). Bioremediation, biostimulation and bioaugmention: a review. International Journal of Environmental Bioremediation \& Biodegradation, 3(1), 28-39.

Affonso, R. S., Rennó, M. N., Slana, G. B., \& Franca, T. C. (2012). Aspectos químicos e biológicos do óleo essencial de cravo da índia. Revista Virtual de Química, 4(2), 146-161.

Arthur, E. L., Rice, P. J., Rice, P. J., Anderson, T. A., Baladi, S. M., Henderson, K. L., \& Coats, J. R. (2005). Phytoremediation-an overview. Critical Reviews in Plant Sciences, 24(2), 109-122.

Bahmani, M., \& Schmidt, O. (2018). Plant essential oils for environment-friendly protection of wood objects against fungi. Maderas. Ciencia y tecnología, 20(3), 325-332.

Bayala, B., Bassole, I. H. N., Gnoula, C., Nebie, R., Yonli, A., Morel, L., \& Simpore, J. (2014). Chemical composition, antioxidant, anti-inflammatory and anti-proliferative activities of essential oils of plants from Burkina Faso. PLoS one, 9(3), e92122.

Biondi, C. M., Nascimento, C. W. A. D., Fabricio Neta, A. D. B., \& Ribeiro, M. R. (2011). Teores de Fe, Mn, Zn, Cu, Ni e Co em solos de referência de Pernambuco. Revista Brasileira de Ciência do Solo, 35(3), 1057-1066.

Bizzo, H. R., Hovell, A. M. C., \& Rezende, C. M. (2009). Óleos essenciais no Brasil: aspectos gerais, desenvolvimento e perspectivas. Química Nova, 32(3), 588-594.

Bouyahya, A., Jamal, A., Edaoudi, F., Et-Touys, A., Bakri, Y., \& Dakka, N. (2016). Origanum compactum Benth: a review on phytochemistry and pharmacological properties. Medicinal and Aromatic Plants, 5(4), 1-6.

Brasil. Ministério da Saúde. Vigilância em Saúde (2014). Vigisolo. Dados e Acompanhamento das Populações. Brasília: Ministério da Saúde. <http://portalms.saude.gov.br/vigilancia-em-saude/vigilancia-ambiental/vigipeq/vigisolo/dados-e-acompanhamento-das-populacoes>.

Brito, G. C. B., \& Vasconcelos, F. C. W. (2012). A gestão de áreas contaminadas em Minas Gerais: o licenciamento como instrumento preventivo. Revista de Gestão Social e Ambiental-RGSA, 6(2), 19-32.

Carson, C. F., Hammer, K. A., \& Riley, T. V. (2006). Melaleuca alternifolia (tea tree) oil: a review of antimicrobial and other medicinal properties. Clinical Microbiology Reviews, 19(1), 50-62.

Castro, R. D. D., \& Lima, E. D. O. (2010). Atividade antifúngica in vitro do óleo essencial de Eucalyptus globulus L. sobre Candida spp. Revista de Odontologia UNESP, 179-184.

Chagas, A. C. D. S., Passos, W. M., Prates, H. T., Leite, R. C., Furlong, J., \& Fortes, I. C. P. (2002). Efeito acaricida de óleos essenciais e concentrados emulsionáveis de Eucalyptus spp em Boophilus microplus. Brazilian Journal of Veterinary Research and Animal Science, $39(5), 247-253$.

Contrucci, B. A., Silva, R., Junior, R. A., \& Kozusny-Andreani, D. I. (2019). Efeito de óleos essenciais sobre bactérias gram-negativas isoladas de alimentos. Ensaios em Ciências Biológicas, Agrárias e da Saúde, 23(3), 180-184.

Craveiro, A. A., \& Queiroz, D. C. (1993). Óleos essenciais e química fina. Química nova, 16(3), 224-228.

Danh, L. T., Truong, P., Mammucari, R., \& Foster, N. (2011). Effect of calcium on growth performance and essential oil of vetiver grass (Chrysopogon zizanioides) grown on lead contaminated soils. International journal of phytoremediation, 13(sup1), 154-165.

EEA (European Environment Agency). A decade of industrial pollution data. 2019. Disponível em: Environmental pressures of heavy metal releases from Europe's industry — European Environment Agency (europa.eu) Acesso em 30/03/2021.

Elallem, K. A., Sobeh, M., Boularbah, A., \& Yasri, A. (2020). Chemically degraded soil rehabilitation process using medicinal and aromatic plants. Environmental Science and Pollution Research, 1-21.

Ferreira, R. M., Lofrano, F. C., \& Morita, D. M. (2020). Remediação de áreas contaminadas: uma avaliação crítica da legislação brasileira. Engenharia Sanitaria e Ambiental, 25(1), 115-125.

Gonçalves Jr, A. C. (2013). Descontaminação e monitoramento de águas e solos na região amazônica utilizando materiais adsorventes alternativos, visando remoção de metais pesados tóxicos e pesticidas. Inclusão Social, 6(2).

Jain, S., Khare, P., Mishra, D., Shanker, K., Singh, P., Singh, R. P., \& Baruah, B. P. (2020). Biochar aided aromatic grass [Cymbopogon martini (Roxb.) Wats.] vegetation: A sustainable method for stabilization of highly acidic mine waste. Journal of Hazardous Materials, $390,121799$. 
Jiménez-Reyes, M. F., Carrasco, H., Olea, A. F., \& Silva-Moreno, E. (2019). Natural compounds: A sustainable alternative to the phytopathogens control. Journal of the Chilean Chemical Society, 64(2), 4459-4465.

Júnior, D. G., Paschoalin, L. F. C., de Oliveira, A. P., \& Botezelli, L. Barreiras à implementação da remediação ambiental no Brasil: aspectos gerais relacionados a minas de urânio. Research, Society and Development, 10(1), e19310110806-e19310110806.

Knaak, N., \& Fiuza, L. M. (2010). Potencial dos óleos essenciais de plantas no controle de insetos e microrganismos. Neotropical Biology \& Conservation, $5(2)$.

Koul, O., Walia, S., \& Dhaliwal, G. S. (2008). Essential oils as green pesticides: potential and constraints. Biopesticides international, 4(1), 63-84.

Machado, B. F. M. T., \& Junior, A. F. (2011). Óleos essenciais: aspectos gerais e usos em terapias naturais. Cadernos Acadêmicos, 3(2), 105-127.

Marques, M., Aguiar, C. R. C., \& Silva, J. J. L. S. D. (2011). Desafios técnicos e barreiras sociais, econômicas e regulatórias na fitorremediação de solos contaminados. Revista Brasileira de Ciência do Solo, 35(1), 1-11.

Martins, G. D. S. O., Zago, H. B., Costa, A. V., Araujo Junior, L. M. D., \& Carvalho, J. R. D. (2017). Chemical composition and toxicity of Citrus essential oils on Dysmicoccus brevipes (Hemiptera: Pseudococcidae). Revista Caatinga, 30(3), 811-817.

Mikola, T. V. Z., Potenza, M. R., Reis, F. C., Silva, V. C. D., Sato, M. E., \& Sakita, M. N. (2017). Evaluation of essential oils of Eucalyptus spp. for the control of the subterranean termite Coptotermes gestroi (Wasman) 1. Revista Árvore, 41(2).

MMA. Ministério do Meio Ambiente. Programa nacional de recuperação de áreas contaminadas: agenda nacional de qualidade ambiental urbana [livro eletrônico] Brasília: MMA 2020.

Najem, M., Bammou, M., Bachiri, L., Bouiamrine, E. H., Ibijbijen, J., \& Nassiri, L. (2020). Ruta chalepensis L. Essential Oil Has a Biological Potential for a Natural Fight against the Pest of Stored Foodstuffs: Tribolium castaneum Herbst. Evidence-Based Complementary and Alternative Medicine, $2020,1-11$.

Okoh, O. O., Sadimenko, A. P., \& Afolayan, A. J. (2010). Comparative evaluation of the antibacterial activities of the essential oils of Rosmarinus officinalis L. obtained by hydrodistillation and solvent free microwave extraction methods. Food Chemistry, 120(1), 308-312.

Pandey, J., Chand, S., Pandey, S., \& Patra, D. D. (2015). Palmarosa [Cymbopogon martinii (Roxb.) Wats.] as a putative crop for phytoremediation, in tannery sludge polluted soil. Ecotoxicology and environmental safety, 122, 296-302.

Pandey, J., Verma, R. K., \& Singh, S. (2019). Suitability of aromatic plants for phytoremediation of heavy metal contaminated areas: a review. International Journal of Phytoremediation, 21(5), 405-418.

Patel, A., \& Patra, D. D. (2014). Influence of heavy metal rich tannery sludge on soil enzymes vis-à-vis growth of Tagetes minuta, an essential oil bearing crop. Chemosphere, 112, 323-332.

Pellegrini, M., Ricci, A., Serio, A., Chaves-López, C., Mazzarrino, G., D’Amato, S., ... \& Paparella, A. (2018). Characterization of essential oils obtained from Abruzzo autochthonous plants: Antioxidant and antimicrobial activities assessment for food application. Foods, 7(2), 19.

Santos, A. L. P., Ruas de Lima, G. W., \& Pedriali Moraes, C. A. (2019). Estudo do potencial do óleo essencial de Lavandula angustiofolia L. como antimicrobiano. InterfacEHS, 14(1).

Pérez, A.P., Eugenio, N.R. Status of local soil contamination in Europe: revision of the indicator "Progress in the management contaminated sites in Europe. Luxemburgo: Publications Office of the European Union, 2018.

Pibiri, M. C., Goel, A., Vahekeni, N., \& Roulet, C. A. (2006). Indoor air purification and ventilation systems sanitation with essential oils. International Journal of Aromatherapy, 16(3-4), 149-153.

Pinto, D. A., Mantovani, E. C., Melo, E. D. C., Sediyama, G. C., \& Vieira, G. H. S. (2014). Produtividade e qualidade do óleo essencial de capim-limão, Cymbopogon citratus, DC., submetido a diferentes lâminas de irrigação. Revista Brasileira de Plantas Medicinais, 16(1), 54-61.

Puvača, N., Lika, E., Cocoli, S., Shtylla Kika, T., Bursić, V., Vuković, G., ... \& Cara, M. (2020). Use of tea tree essential oil (Melaleuca alternifolia) in laying hen's nutrition on performance and egg fatty acid profile as a promising sustainable organic agricultural tool. Sustainability, 12(8), 3420.

Ramborger, B. P., Paz, M. E. G., Kieling, K. M. C., Carriço, M. R. S., de Paula Gollino, G., Costa, M. T., ... \& Roehrs, R. (2021). Toxicological parameters of aqueous residue after using Plectranthus neochilus for 2, 4-D phytoremediation. Chemosphere, 270, 128638.

Rienth, M., Crovadore, J., Ghaffari, S., \& Lefort, F. (2019). Oregano essential oil vapour prevents Plasmopara viticola infection in grapevine (Vitis vinifera) and primes plant immunity mechanisms. PLoS One, 14(9), e0222854.

Rodrigues, P. T. A., \& Orlandelli, R. C. (2018). Plantas como Ferramentas para a Remediação Ambiental: uma Revisão da Literatura. UNICIÊNCIAS, 22(1), $38-44$.

Rogawansamy, S., Gaskin, S., Taylor, M., \& Pisaniello, D. (2015). An evaluation of antifungal agents for the treatment of fungal contamination in indoor air environments. International Journal of Environmental Research and Public Health, 12(6), 6319-6332.

Sangwan, N. S., Farooqi, A. H. A., Shabih, F., \& Sangwan, R. S. (2001). Regulation of essential oil production in plants. Plant Growth Regulation, 34(1), 321.

Scalvenzi, L., Radice, M., Toma, L., Severini, F., Boccolini, D., Bella, A., \& Di Luca, M. (2019). Larvicidal activity of Ocimum campechianum, Ocotea quixos and Piper aduncum essential oils against Aedes aegypti. Parasite, 26, 1-12. 
Research, Society and Development, v. 10, n. 7, e3210716146, 2021

(CC BY 4.0) | ISSN 2525-3409 | DOI: http://dx.doi.org/10.33448/rsd-v10i7.16146

Sengun, I. Y., Senturk, S., Gul, S., \& Kilic, G. (2020). Potential of Essential Oil Combinations for Surface and Air Disinfection. Letters in Applied Microbiology, 72, 526-534.

Sienkiewicz, M., Głowacka, A., Kowalczyk, E., Wiktorowska-Owczarek, A., Jóźwiak-Bębenista, M., \& Łysakowska, M. (2014). The biological activities of cinnamon, geranium and lavender essential oils. Molecules, 19(12), 20929-20940.

Soylu, E. M., Kurt, Ş., \& Soylu, S. (2010). In vitro and in vivo antifungal activities of the essential oils of various plants against tomato grey mould disease agent Botrytis cinerea. International Journal of Food Microbiology, 143(3), 183-189.

Tian, Q., Zhou, W., Cai, Q., Ma, G., \& Lian, G. (2021). Concepts, processing, and recent developments in encapsulating essential oils. Chinese Journal of Chemical Engineering, 30, 255-271.

Ultra, V. U. (2020). Growth and yield of lemongrass (Cymbopogon citratus) in fly ash with nutrient amendments and Mycorrhiza for three-ratoon period. International Journal of Phytoremediation, 22(14), 1551-1561.

USEPA (United States Environmental Protection Agency). Cleaning Up the Nation's Waste Sites: Markets and Technology Trends. Washington: EPA, 2004. Vasconcellos, M. C., Pagliuso, D., \& Sotomaior, V. S. (2012). Fitorremediação: Uma proposta de descontaminação do solo. Estudos de Biologia, 34(83).

Van Eck, N. J., Waltman, L. Software survey: VOSviewer, a computer program for bibliometric mapping. Scientometrics, v. 84. n. 2, p. 523-538, 2010.

Vaseeharan, B., Sivalingam, M., \& Palaniappan, R. (2013). Inhibitory activity of essential oils from medicinal plants against Pseudomonas sp. isolated from aquatic environments. Aquaculture Research, 45(1), 97-105.

Vidali, M. (2001). Bioremediation. an overview. Pure and Applied Chemistry, 73(7), 1163-1172.

Vieira-Brock, P. L., Vaughan, B. M., \& Vollmer, D. L. (2017). Comparison of antimicrobial activities of natural essential oils and synthetic fragrances against selected environmental pathogens. Biochimie Open, 5, 8-13.

Vinu, R., \& Madras, G. (2010). Environmental remediation by photocatalysis. Journal of the Indian Institute of Science, 90(2), 189-230.

Vitoratos, A., Bilalis, D., Karkanis, A., \& Efthimiadou, A. (2013). Antifungal activity of plant essential oils against Botrytis cinerea, Penicillium italicum and Penicillium digitatum. Notulae Botanicae Horti Agrobotanici Cluj-Napoca, 41(1), 86-92.

Volpe, H. X., Fazolin, M., Garcia, R. B., Magnani, R. F., Barbosa, J. C., \& Miranda, M. P. (2016). Efficacy of essential oil of Piper aduncum against nymphs and adults of Diaphorina citri. Pest Management Science, 72(6), 1242-1249.

Youssef, N. A. (2021). Changes in the morphological traits and the essential oil content of sweet basil (Ocimum basilicum L.) as induced by cadmium and lead treatments. International Journal of Phytoremediation, 23(3), 291-299. 